\title{
Singular Points of the Wigner Caustic and Affine Equidistants of Planar Curves
}

\author{
Wojciech Domitrz ${ }^{1}\left[0 \cdot\right.$ Michał Zwierzyński $^{1}$
}

Received: 26 October 2018 / Accepted: 29 March 2019 / Published online: 3 April 2019

(c) The Author(s) 2019

\begin{abstract}
In this paper we study singular points of the Wigner caustic and affine $\lambda$-equidistants of planar curves based on shapes of these curves. We generalize the Blaschke-Süss theorem on the existence of antipodal pairs of a convex curve.
\end{abstract}

Keywords Wigner caustic · Affine equidistants - Singularities · Planar curves · Semiclassical dynamics

Mathematics Subject Classification 53A04 · 53A15 - 58K05 -81Q20

\section{Introduction}

The famous Blaschke-Süss theorem states that there are at least three pairs of antipodal pairs on an oval (Góźdź 1990; Laugwitz 1965). We recall that a pair of points on an oval is an antipodal pair if the tangent lines are parallel at these points and curvatures are equal. The Wigner caustic is a locus of midpoints of chords connecting points on a curve with parallel tangent lines (Berry 1977; Domitrz et al. 2013; Domitrz and de M Rios 2014; Domitrz and Zwierzyński 2016; Ozorio de Almeida and Hannay 1982). The singular points of the Wigner caustic of an oval come exactly from the antipodal pairs. The Wigner caustic was first considered by Berry in Berry (1977) on the study of Wigner's phase-space representation of quantum states. Recently many interesting properties of the Wigner caustic were investigated. For instance the typical

The work of W. Domitrz and M. Zwierzyński was partially supported by NCN Grant no. DEC-2013/11/B/ST1/03080.

$凶$ Wojciech Domitrz

domitrz@mini.pw.edu.pl

Michał Zwierzyński

zwierzynskim@mini.pw.edu.pl

1 Faculty of Mathematics and Information Science, Warsaw University of Technology, ul. Koszykowa 75, 00-662 Warszawa, Poland 
behavior of convex bodies in $\mathbb{R}^{2}$ were studied by Schneider using the middle hedgehog (Schneider 2016, 2017) which is a natural generalization of the Wigner caustic for non-smooth ovals. Furthermore the absolute value of the oriented area of the Wigner caustic improves the classical isoperimetric inequality for ovals (Zwierzyński 2016a, b) and improves the isoperimetric defect in the reverse isoperimetric inequality (Cufi et al. 2018). Let us also notice the use of this set in the affine geometry. It leads to the construction of bi-dimensional improper affine spheres (Craizer et al. 2015). The Wigner caustic is an example of an affine $\lambda$-equidistant, which is the locus of points dividing chords connecting points on $M$ with parallel tangent lines in a fixed ratio $\lambda$ (Domitrz et al. 2014, 2016; Domitrz and Zwierzyński 2016; Zakalyukin 1995).

In this paper we study singular points of Wigner caustic and affine equidistants of a planar curve. We prove theorems on existence of singular points based on the shape of the curve. In particular we find a generalization of the Blaschke-Süss theorem (see Theorem 3.2).

In Sect. 2 we briefly sketch the known results on the Wigner caustic and affine equidistants. Then we proceed with the study of tangent lines and curvatures of affine equidistants and thanks to it we obtain many global results concerning properties of affine equidistants. Among other things we derive a formula for the number of their inflexion points, we prove that it is possible to reconstruct the original convex curve from any affine equidistants except the Wigner caustic and we show that any equidistant, except the Wigner caustic, (for a generic $\lambda$ ) of a generic closed convex curve has an even number of cusp singularities.

In Sect. 3 theorems on existence of singularities of affine equidistants and the Wigner caustic are proved and some applications are indicated.

The methods used in this paper can be also applied to study singular points of the secant caustic (Domitrz et al. 2018).

\section{Properties of the Wigner Caustic and Affine Equidistants}

A smooth parameterized curve $M$ on the affine plane $\mathbb{R}^{2}$ is the image of a $C^{\infty}$ smooth map $f: I \rightarrow \mathbb{R}^{2}$, where $I$ is an open interval in $\mathbb{R}$. The image of a $C^{\infty}$ map $f: S^{1} \rightarrow \mathbb{R}^{2}$ is called a smooth closed curve. The map $f$ is called the parameterization of $M$.

A point $f\left(s_{0}\right)$ is called a regular point of $M$ if $f^{\prime}\left(s_{0}\right) \neq 0$, where $f$ is the parameterization of $M$. If every point of a smooth curve is a regular point, then the curve is called regular. If a point $f\left(s_{0}\right)$ is not a regular point (i.e. $\left.f^{\prime}\left(s_{0}\right)=0\right)$, then we call it a singular point. A curve is called singular if it has a singular point.

A curve with no intersection points is called simple. A regular simple closed curve is a convex curve if its curvature does not vanish.

Definition 2.1 A pair of different points $a, b$ in $M$ is called a parallel pair if the lines $T_{a} M$ and $T_{b} M$ are parallel.

Definition 2.2 An affine $\lambda$-equidistant is the following set

$$
\mathrm{E}_{\lambda}(M)=\{\lambda a+(1-\lambda) b \mid a, b \text { is a parallel pair of } M\} .
$$


(a)

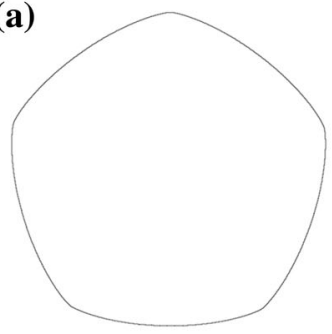

(d)

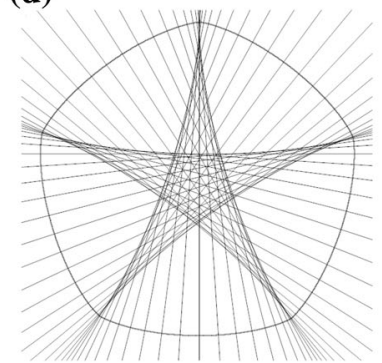

(b)

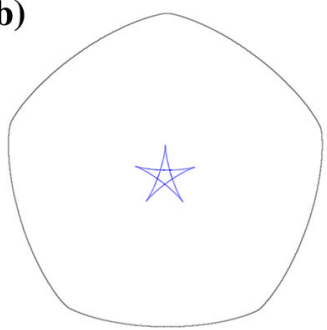

(e)

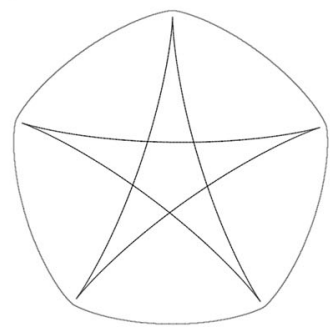

(c)

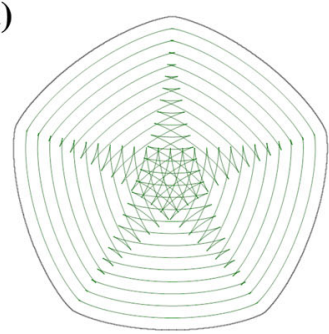

(f)

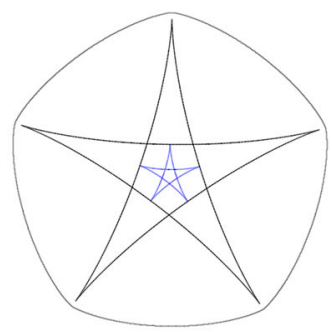

Fig. 1 a A curve $M, \mathbf{b} E_{\frac{1}{2}}(M), \mathbf{c} E_{\lambda}(M)$ for $\lambda=\frac{k}{26}$ for $k=1,2, \ldots, 13, \mathbf{d}$ lines joining parallel pairs of $M, \mathbf{e} \operatorname{CSS}(M), \mathbf{f} E_{\frac{1}{2}}(M)$ and $\operatorname{CSS}(M)$

The Wigner caustic of $M$ is $\mathrm{E}_{\frac{1}{2}}(M)$.

Definition 2.3 The Centre Symmetry Set of $M(\operatorname{CSS}(M))$ is the envelope of lines joining parallel pairs of $M$.

Let us briefly sketch the known results on the geometry of affine $\lambda$-equidistants and the Centre Symmetry Set (see Fig. 1). Let $M$ be a generic convex curve. Then $\operatorname{CSS}(M)$, $E_{\frac{1}{2}}(M)$ and $E_{\lambda}(M)$ for a generic $\lambda$ are smooth closed curves which can have only cusp singularities (Berry 1977; Giblin and Holtom 1999; Giblin and Zakalyukin 2005; Janeczko 1996). Furthermore regular parts of CSS $(M)$ are formed by cusp singularities of $\mathrm{E}_{\lambda}(M)$ (Giblin and Zakalyukin 2005). Both: the number of cusp singularities of $E_{\frac{1}{2}}(M)$ and the number of cusp singularities of $\operatorname{CSS}(M)$, are odd and not smaller than 3 (Berry 1977; Giblin and Holtom 1999; Góźdź 1990) and the number of cusp singularities of $E_{\frac{1}{2}}(M)$ is not greater than the number of cusp singularities of $\operatorname{CSS}(M)$ (Domitrz and de $\mathrm{M}$ Rios 2014). We will show that the number of cusps of $\mathrm{E}_{\lambda}(M)$ for a generic $\lambda \neq \frac{1}{2}$ is even (Theorem 2.16). Bifurcations of affine $\lambda$-equidistants were studied in details in Giblin and Reeve (2015), Giblin et al. (2009), Janeczko et al. (2014). The global geometry of the Wigner caustic and other affine $\lambda$-equidistants were studied in Domitrz and Zwierzyński (2016). The geometry of an affine extended wave front, i.e. the set $\bigcup_{\lambda \in[0,1]}\{\lambda\} \times E_{\lambda}(M)$ was studied in Domitrz and Zwierzyński (2018). Let $p$ be a inflexion point of $M$. Then $\operatorname{CSS}(M)$ is tangent to this inflexion point and has an endpoint there. The set $\mathrm{E}_{\lambda}(M)$ for $\lambda \neq \frac{1}{2}$ has an inflexion point at $p$ (as the limit point) and is tangent to $M$ at $p$. The Wigner caustic is tangent to $M$ at 
$p$ too and it has an endpoint there. The Wigner caustic and the Centre Symmetry Set approach $p$ from opposite sides (Berry 1977; Domitrz et al. 2013; Giblin and Holtom 1999; Giblin et al. 2009). The geometry of this branch of the Wigner caustic, which is called the Wigner caustic on shell, is studied in Domitrz and Zwierzyński (2016).

Let us denote by $\kappa_{M}(p)$ the signed curvature of $M$ at $p$.

If $a, b$ is a parallel pair of $M$ such that local parameterizations of $M$ nearby points $a$ and $b$ are in opposite directions and $\kappa_{M}(a)+\kappa_{M}(b) \neq 0$, then the point $\frac{\kappa_{M}(a) \cdot a+\kappa_{M}(b) \cdot b}{\kappa_{M}(a)+\kappa_{M}(b)}$ belongs to $\operatorname{CSS}(M)$ (Giblin and Holtom 1999).

By direct calculations we get the following lemma.

Lemma 2.4 Let $M$ be a closed regular curve. Let $a, b$ be a parallel pair of $M$, such that $M$ is parameterized at $a$ and $b$ in opposite directions and $\kappa_{M}(b) \neq 0$. Let $p=$ $\lambda a+(1-\lambda) b$ be a regular point of $E_{\lambda}(M)$ and let $q=\frac{\kappa_{M}(a) \cdot a+\kappa_{M}(b) \cdot b}{\kappa_{M}(a)+\kappa_{M}(b)}$ be a regular point of $\operatorname{CSS}(M)$.

Then

(i) the tangent line to $E_{\lambda}(M)$ at $p$ is parallel to the tangent lines to $M$ at $a$ and $b$.

(ii) the curvature of $E_{\lambda}(M)$ at $p$ is equal to

$$
\kappa_{E_{\lambda}(M)}(p)=\frac{\kappa_{M}(a)\left|\kappa_{M}(b)\right|}{\left|\lambda \kappa_{M}(b)-(1-\lambda) \kappa_{M}(a)\right|} .
$$

(iii) the curvature of $\operatorname{CSS}(M)$ at $q$ is equal to

$$
\kappa_{C S S(M)}(q)=\operatorname{sgn}\left(\kappa_{M}(b)\right) \cdot \frac{\left(\kappa_{M}(a)+\kappa_{M}(b)\right)^{3}}{\left|\kappa_{M}^{2}(b) \kappa_{M}^{\prime}(a)-\kappa_{M}^{2}(a) \kappa_{M}^{\prime}(b)\right|} \cdot \frac{\operatorname{det}(a-b, \mathbb{t}(a))}{|a-b|^{3}},
$$

where $\mathbb{t}(a)$ is a tangent unit vector field compatible with the orientation of $M$ at $a$.

Lemma 2.4(ii-iii) implies the following propositions.

Proposition 2.5 (Giblin and Zakalyukin 2005) Let $a, b$ be a parallel pair of a generic regular curve $M$, such that $M$ is parameterized at a and $b$ in opposite directions and $\kappa_{M}(b) \neq 0$. Then the point $\lambda a+(1-\lambda) b$ is a singular point of $E_{\lambda}(M)$ if and only if $\frac{\kappa_{M}(a)}{\kappa_{M}(b)}=\frac{\lambda}{1-\lambda}$.

Proposition 2.6 (Domitrz and Zwierzyński 2016) Let $a, b$ be a parallel pair of $a$ generic regular closed curve $M$ and let $\lambda \neq 0,1$. Then $\lambda a+(1-\lambda) b$ and $(1-\lambda) a+\lambda b$ are inflexion points of $E_{\lambda}(M)$ if and only if one of the points $a, b$ is an inflexion point of $M$.

Corollary 2.7 If $M$ is a convex curve, then $E_{\lambda}(M)$ for $\lambda \in \mathbb{R}$ and $\operatorname{CSS}(M)$ have no inflexion points.

Let $\tau_{p}$ denote the translation by a vector $p \in \mathbb{R}^{2}$.

Definition 2.8 A curve $M$ is curved in the same side at $a$ and $b$ (resp. curved in the different sides), where $a, b$ is a parallel pair of $M$, if the center of curvature of $M$ at $a$ and the center of curvature of $\tau_{a-b}(M)$ at $a=\tau_{a-b}(b)$ lie on the same side (resp. on the different sides) of the tangent line to $M$ at $a$. 
(a)
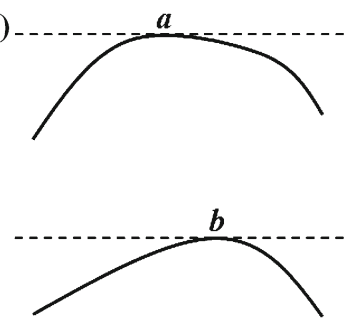

(b)
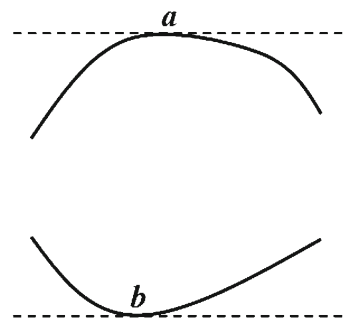

Fig. 2 a A curve curved in the same side at a parallel pair, $\mathbf{b}$ a curve curved in the different sides at a parallel pair

Proposition 2.9 (Domitrz and Zwierzyński 2016)

(i) If $M$ is curved in the same side at a parallel pair $a, b$, then $\lambda a+(1-\lambda) b$ is $a$ $C^{\infty}$ regular point of $E_{\lambda}(M)$ for $\lambda \in(0,1)$.

(ii) If $M$ is curved in the different sides at a parallel pair $a, b$, then $\lambda a+(1-\lambda) b$ is $a C^{\infty}$ regular point of $E_{\lambda}(M)$ for $\lambda \in(-\infty, 0) \cup(1, \infty)$.

Definition 2.10 The tangent line of $\mathrm{E}_{\lambda}(M)$ at a cusp point $p$ is the limit of a sequence of 1-dimensional vector spaces $T_{q_{n}} M$ in $\mathbb{R} P^{1}$ for any sequence $q_{n}$ of regular points of $\mathrm{E}_{\lambda}(M)$ converging to $p$.

By Lemma 2.4(i) the tangent line to $\mathrm{E}_{\lambda}(M)$ at the cusp point $\lambda a+(1-\lambda) b$ is parallel to the tangent lines to $M$ at $a$ and $b$.

Definition 2.11 Let $\lambda \neq \frac{1}{2}$. The normal vector field to $\mathrm{E}_{\lambda}(M)$ at the point $\lambda a+(1-\lambda) b$ is equal to the normal vector field to $M$ at the point $a$.

Remark 2.12 If $M$ is convex we have the well defined continuous normal vector field on the double covering $M$ of $\mathrm{E}_{\frac{1}{2}}(M)$, namely: $M \ni a \mapsto \frac{a+b}{2} \in \mathrm{E}_{\frac{1}{2}}(M)$.

Let us notice that the continuous normal vector field to $\mathrm{E}_{\lambda}(M)$ at regular and cusp points is perpendicular to the tangent line to $\mathrm{E}_{\lambda}(M)$. Using this fact and the above definition we define the rotation number in the following way.

Definition 2.13 The rotation number of a smooth curve $M$ with well defined continuous normal vector field is the rotation number of this vector field.

Moreover by Lemma 2.4(i) we can easily get the next two propositions.

Proposition 2.14 Let $M$ be a generic regular closed curve in $\mathbb{R}^{2}$. If $l$ is a bitangent line to $M$ at points $a, b$, then $E_{\lambda}(M)$ is tangent to $l$ at points $\lambda a+(1-\lambda) b$ and $(1-\lambda) a+\lambda b$.

If $M$ is a convex curve, then for any line $l$ in $\mathbb{R}^{2}$ there exist exactly two points $a, b \in M$ in which the tangent lines to $M$ at $a$ and $b$ are parallel to $l$. Also there exists exactly one parallel pair $a, b$ of $M$ such that chord passing through $a$ and $b$ is parallel to $l$. Therefore we get the following proposition. 
Fig. 3 An ordinary cusp singularity with a continuous normal vector field. Vectors in upper regular component of a curve are directed outside the cusp, others are directed inside the cusp

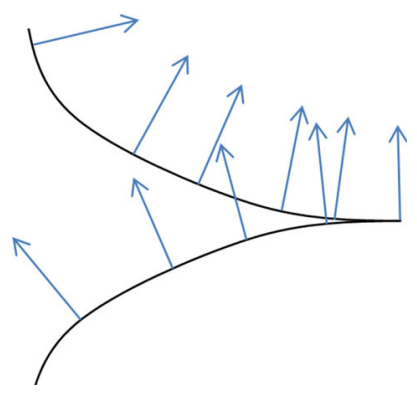

Proposition 2.15 Let $M$ be a generic convex curve. Then for any line l in $\mathbb{R}^{2}$

(i) there exists exactly one point $p$ in $E_{\frac{1}{2}}(M)$ such that the tangent line to $E_{\frac{1}{2}}(M)$ at $p$ is parallel to $l$.

(ii) there exist exactly two different points $p_{1}, p_{2}$ in $E_{\lambda}(M)$ for $\lambda \neq \frac{1}{2}$, such that the tangent lines to $E_{\lambda}(M)$ at $p_{1}$ and $p_{2}$ are parallel to $l$.

(iii) there exists exactly one point $p$ in $\operatorname{CSS}(M)$ such that the tangent line to $\operatorname{CSS}(M)$ at $p$ is parallel to $l$ (Giblin and Holtom 1999).

Theorem 2.16 Let $M$ be a generic convex curve. Then the number of cusps of $E_{\lambda}(M)$ for a generic $\lambda \neq \frac{1}{2}$ is even and the number of cusps of $E_{\frac{1}{2}}(M)$ is odd.

Proof For a generic convex curve $M, \mathrm{E}_{\frac{1}{2}}(M)$ has got only cusp singularities and the same statement holds for $\mathrm{E}_{\lambda}(M)$ for a generic $\lambda \neq \frac{1}{2}$. Without loss of generality we may assume that $M$ is positively oriented.

Let $P: M \rightarrow M$ maps a point $a$ to the point $b \neq a$ such that $a, b$ is a parallel pair. This map is well defined on a convex curve $M$. If $f: S^{1} \rightarrow \mathbb{R}^{2}$ is a parameterization of $M$, then $S^{1} \ni s \mapsto \lambda f(s)+(1-\lambda) P(f(s)) \in \mathrm{E}_{\lambda}(M)$ is a parameterization of $\mathrm{E}_{\lambda}(M)$ for $\lambda \neq \frac{1}{2}$ and it is a double covering of $\mathrm{E}_{\frac{1}{2}}(M)$. The normal vector to $\mathrm{E}_{\lambda}(M)$ at $\lambda f(s)+(1-\lambda) P(f(s))$ is the normal vector to $M$ at $f(s)$, so the rotation number of $\mathrm{E}_{\lambda}(M)$ for $\lambda \neq \frac{1}{2}$ is equal to the rotation number of $M$, which is 1 for positively oriented convex curves, and the rotation number of $\mathrm{E}_{\frac{1}{2}}(M)$ is equal to $\frac{1}{2}$.

A continuous normal vector field to the germ of a curve with the cusp singularity is directed outside the cusp on the one of two connected regular components and is directed inside the cusp on the other component as it is illustrated in Fig. 3. Since the rotation number of $\mathrm{E}_{\lambda}(M)$ for $\lambda \neq \frac{1}{2}$ is 1 , the number of cusps of $\mathrm{E}_{\lambda}(M)$ for $\lambda \neq \frac{1}{2}$ is even and the number of cusps of $\mathrm{E}_{\frac{1}{2}}(M)$ is odd because the rotation number of $\mathrm{E}_{\frac{1}{2}}(M)$ is equal to $\frac{1}{2}$. The last statement has been proved by M. Berry in Berry (1977) using a different method.

In Berry (1977), Laugwitz (1965) it is proved by analytical methods that the number of cusp singularities of the Wigner caustic of a generic convex curve is odd and not smaller than 3 . We present an elementary proof of this fact for the Wigner caustic and the Centre Symmetry Set. 
Fig. 4 The cusp $\left(p_{c}\right)$, dotted lines are parallel

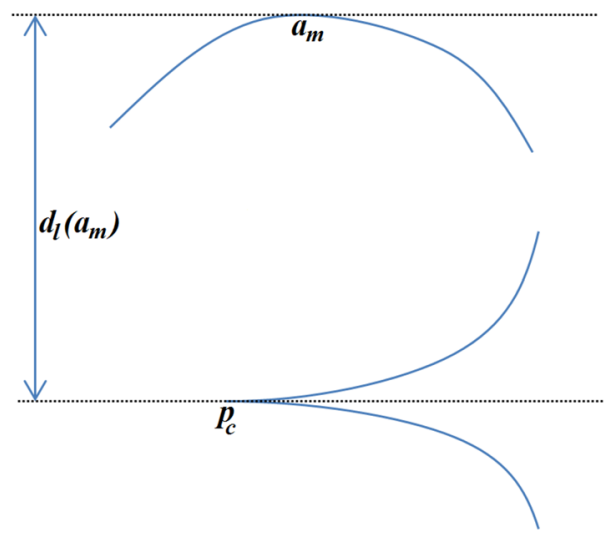

Proposition 2.17 Let $M$ be a generic convex curve. Then the number of cusps of $C S S(M)$ and $E_{\frac{1}{2}}(M)$ is not smaller than 3 .

Proof The number of cusps of the Wigner caustic is odd (see Theorem 2.16 or Berry 1977). Let us assume that the Wigner caustic has got exactly one cusp. Let $p_{c}$ be the cusp point. Let us consider the line $l$, which is tangent to $\mathrm{E}_{\frac{1}{2}}(M)$ at $p_{c}$. Let $d_{l}(a)$ be the distance between a point $a \in \mathrm{E}_{\frac{1}{2}}(M)$ and $l$. Since $d_{l}\left(p_{c}\right)=0$ and every point $a \in \mathrm{E}_{\frac{1}{2}}(M)$ such that $a \neq p_{c}$ is a regular point of $\mathrm{E}_{\frac{1}{2}}(M)$, there exists a point $a_{m}$ such that $d_{l}\left(a_{m}\right)$ is a maximum value of $d_{l}$ (see Fig. 4). Then the tangent line to the Wigner caustic at $a_{m}$ is parallel to $l$, which is impossible by Proposition 2.15(i).

By Proposition 2.15(iii), the proof for $\operatorname{CSS}(M)$ is similar.

Proposition 2.18 Let $M$ be a generic convex curve.

(i) If $\lambda \neq \frac{1}{2}$, then

$$
E_{\delta}\left(E_{\lambda}(M)\right)=E_{\delta(1-\lambda)+\lambda(1-\delta)}(M)
$$

(ii) If $\lambda \neq \frac{1}{2}, \delta \neq \frac{1}{2}$, then

$$
\operatorname{CSS}\left(E_{\lambda}(M)\right)=\operatorname{CSS}\left(E_{\delta}(M)\right)
$$

Proof Let $a, b$ be a parallel pair of $M$. By Theorem 2.4(i) $p=\lambda a+(1-\lambda) b$, $q=(1-\lambda) a+\lambda b$ is a parallel pair of $\mathrm{E}_{\lambda}(M)$ and by Proposition 2.15 there are no more points on $\mathrm{E}_{\lambda}(M)$ for which tangent lines at them are parallel to tangent lines at $p, q$. Then $r=\delta p+(1-\delta) q$ is a point of $\mathrm{E}_{\delta}\left(\mathrm{E}_{\lambda}(M)\right)$ and one can get that $r=(1-\Lambda) a+\Lambda b$ where $\Lambda=\delta(1-\lambda)+\lambda(1-\delta)$. Any equidistant of $M$, except the Wigner caustic, has the same collection of chords joining parallel pairs on them, then the Centre Symmetry Set of any equidistant, as an envelope of these lines, is the same set.

By Proposition 2.18 we get the following corollary on reconstruction of the original curve from its affine equidistant and the stability of the Wigner caustic. 
Corollary 2.19 Let $M$ be a generic convex curve. If $\lambda \neq \frac{1}{2}$, then

$$
\begin{aligned}
& M=E_{-\lambda(1-2 \lambda)^{-1}}\left(E_{\lambda}(M)\right)=E_{(1-\lambda)(1-2 \lambda)^{-1}}\left(E_{\lambda}(M)\right), \\
& E_{\frac{1}{2}}\left(E_{\lambda}(M)\right)=E_{\frac{1}{2}}(M) .
\end{aligned}
$$

\section{Existence of a Singular Point of an Affine $\lambda$-Equidistant}

In this section we prove theorems on existence of singularities of affine equidistants and the Wigner caustic. We also present some applications of them.

Definition 3.1 A simple smooth curve $\gamma:\left(s_{1}, s_{2}\right) \rightarrow \mathbb{R}^{2}$ with non-vanishing curvature is called a loop if $\lim _{s \rightarrow s_{1}^{+}} \gamma(s)=\lim _{s \rightarrow s_{2}^{-}} \gamma(s)$. A loop $\gamma$ is called convex if the absolute value of its rotation number is not greater than 1 , otherwise it is called non-convex.

We illustrate examples of loops in Fig. 5.

Theorem 3.2 The Wigner caustic of a loop has a singular point.

Theorem 3.2 follows from the more general, but technical, propositions: Proposition 3.7 in the case of convex loops and Proposition 3.9 in the case of non-convex loops.

Proposition 3.3 Let $\mathcal{F}_{0}, \mathcal{F}_{1}$ be embedded curves with endpoints $p_{0}, q_{0}$ and $p_{1}, q_{1}$, respectively, such that

(i) $p_{0}, p_{1}$ and $q_{0}, q_{1}$ are parallel pairs,

(ii) for every point $a_{1}$ in $\mathcal{F}_{1}$ there exists a point $a_{0}$ in $\mathcal{F}_{0}$ such that $a_{0}, a_{1}$ is a parallel pair,

(iii) $\kappa_{\mathcal{F}_{1}}\left(p_{1}\right), \kappa_{\mathcal{F}_{1}}\left(q_{1}\right)$ and $\kappa_{\mathcal{F}_{0}}\left(a_{0}\right)$ for every $a_{0} \in \mathcal{F}_{0}$ are positive,

(iv) the rotation number of $\mathcal{F}_{0}$ is smaller than $\frac{1}{2}$,

(v) $\mathcal{F}_{0}, \mathcal{F}_{1}$ are curved in the different sides at $p_{0}, p_{1}$ and $q_{0}, q_{1}$.

If $\rho_{\min }$ (resp. $\rho_{\max }$ ) is the minimum (resp. the maximum) of the set $\left\{\frac{\kappa \mathcal{F}_{1}\left(p_{1}\right)}{\kappa_{\mathcal{F}_{0}}\left(p_{0}\right)}, \frac{\kappa_{\mathcal{F}_{1}}\left(q_{1}\right)}{\kappa_{\mathcal{F}_{0}}\left(q_{0}\right)}\right\}$ then the set $E_{\lambda}\left(\mathcal{F}_{0} \cup \mathcal{F}_{1}\right)$ has a singular point for every $\lambda \in\left[\frac{\rho_{\min }}{1+\rho_{\min }}, \frac{\rho_{\max }}{1+\rho_{\max }}\right] \cup\left[1-\frac{\rho_{\max }}{1+\rho_{\max }}, 1-\frac{\rho_{\min }}{1+\rho_{\min }}\right]$.

Fig. 5 a A convex loop $L$ (the dashed line) and $\mathrm{E}_{\frac{1}{2}}(L), \mathbf{b}$ a non-convex loop $L^{2}$ (the dashed line) and $\mathrm{E}_{\frac{1}{2}}(L)$
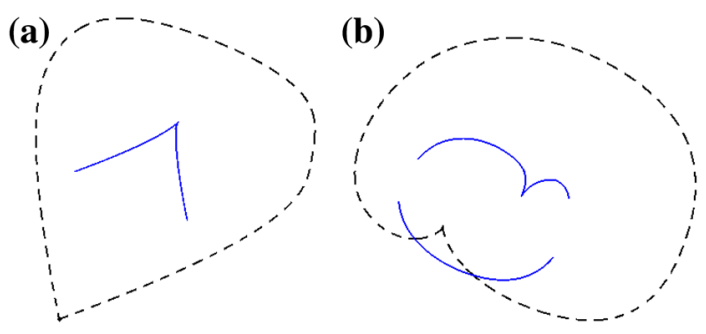
Fig. 6 Arcs satisfying assumptions of Proposition 3.3

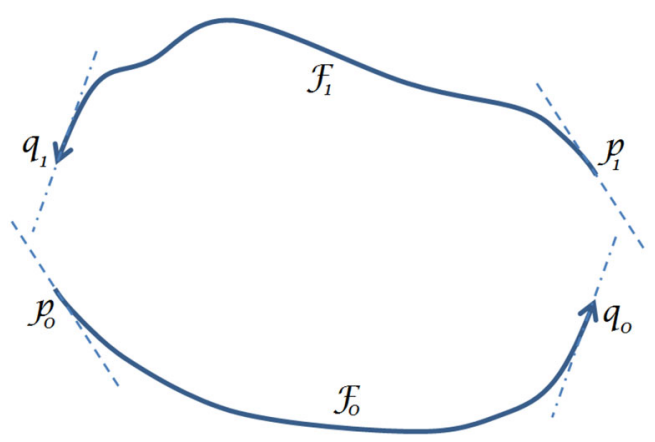

Proof Since $\mathrm{E}_{\lambda}\left(\mathcal{F}_{0} \cup \mathcal{F}_{1}\right)=\mathrm{E}_{1-\lambda}\left(\mathcal{F}_{0} \cup \mathcal{F}_{1}\right)$, let us fix $\lambda$ in $\left[\frac{\rho_{\min }}{1+\rho_{\min }}, \frac{\rho_{\max }}{1+\rho_{\max }}\right]$. Let $g:\left[t_{0}, t_{1}\right] \rightarrow \mathbb{R}^{2}, f:\left[s_{0}, s_{1}\right] \rightarrow \mathbb{R}^{2}$ be the arc length parameterizations of $\mathcal{F}_{0}, \mathcal{F}_{1}$, respectively. From (ii-iii) we get that for every point $a_{1}$ in $\mathcal{F}_{1}$ there exists exactly one point $a_{0}$ in $\mathcal{F}_{0}$ such that $a_{0}, a_{1}$ is a parallel pair. Therefore there exists a function $t:\left[s_{0}, s_{1}\right] \rightarrow\left[t_{0}, t_{1}\right]$ such that

$$
f^{\prime}(s)=-g^{\prime}(t(s))
$$

By the implicit function theorem the function $t$ is smooth and $t^{\prime}(s)=\frac{\kappa \mathcal{F}_{1}(f(s))}{\kappa \mathcal{F}_{0}(g(t(s)))}$. Let $\varrho=\frac{\lambda}{1-\lambda}$. Then $\varrho$ belongs to $\left[\rho_{\min }, \rho_{\max }\right]$. By Darboux Theorem there exists $s \in\left[s_{0}, s_{1}\right]$ such that $t^{\prime}(s)=\varrho$. Then by Proposition 2.5 the set $\mathrm{E}_{\lambda}\left(\mathcal{F}_{0} \cup \mathcal{F}_{1}\right)$ has a singular point for $\lambda=\frac{\varrho}{1+\varrho}$.

Arcs satisfying assumptions of Proposition 3.3 are illustrated in Fig. 6.

By the same argument we get the following result.

Proposition 3.4 Under the assumptions of Proposition 3.3 if we replace (iv) with

(iv) $\mathcal{F}_{0}, \mathcal{F}_{1}$ are curved in the same side at $p_{0}, p_{1}$ and $q_{0}, q_{1}$,

then the set $E_{\lambda}\left(\mathcal{F}_{0} \cup \mathcal{F}_{1}\right)$ has a singular point

- for every $\lambda \in\left[\frac{\rho_{\min }}{\rho_{\min }-1}, \frac{\rho_{\max }}{\rho_{\max }-1}\right] \cup\left[1-\frac{\rho_{\max }}{\rho_{\max }-1}, 1-\frac{\rho_{\min }}{\rho_{\min }-1}\right]$ if $\rho_{\min }>1$,

- for every $\lambda \in\left(-\infty, 1-\frac{\rho_{\max }}{\rho_{\max }-1}\right] \cup\left[\frac{\rho_{\max }}{\rho_{\max }-1}, \infty\right)$ if $\rho_{\min }<1<\rho_{\max }$.

Propositions 3.5 and 3.6 are the limiting versions of Propositions 3.3 and 3.4, respectively.

Proposition 3.5 Let $\mathcal{F}_{0}, \mathcal{F}_{1}$ be embedded curves with endpoints $p_{0}, q_{0}$ and $p_{1}, q_{1}$, respectively, such that

(i) $p_{0}, p_{1}$ and $q_{0}, q_{1}$ are parallel pairs,

(ii) for every point $a_{1}$ in $\mathcal{F}_{1}$ there exists a point $a_{0}$ in $\mathcal{F}_{0}$ such that $a_{0}, a_{1}$ is a parallel pair, 

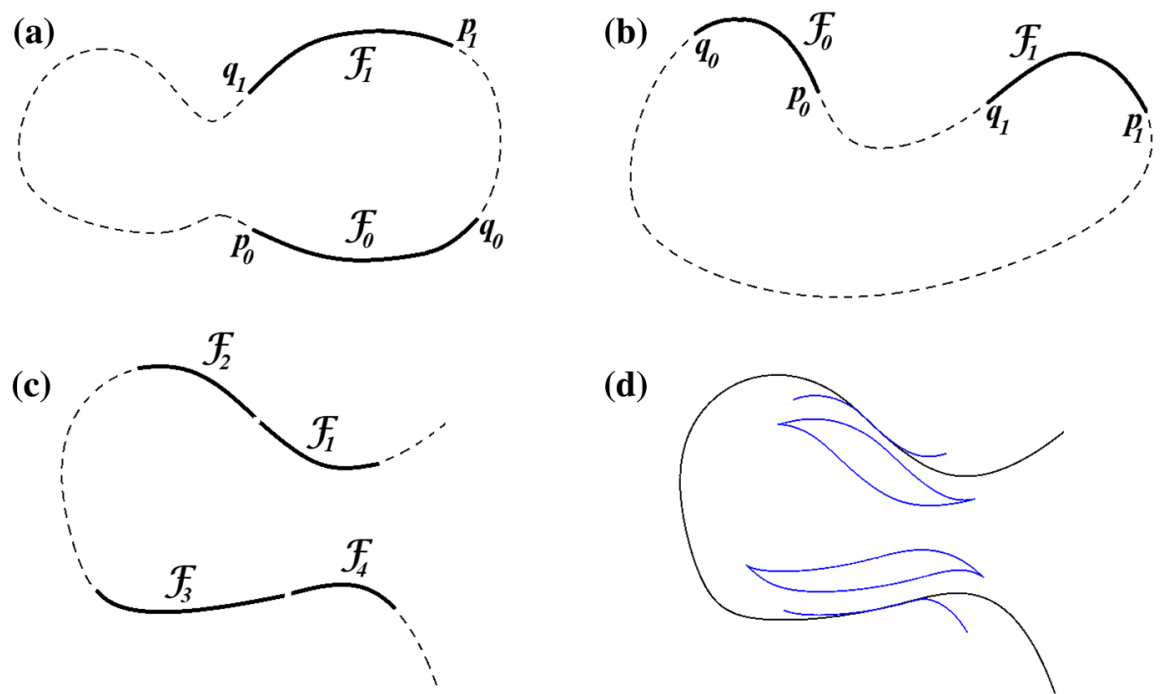

Fig. 7 a Arcs satisfying assumptions of Proposition 3.5, b arcs satisfying assumptions of Proposition 3.6, c arcs $\mathcal{F}_{1}, \mathcal{F}_{4}$ and $\operatorname{arcs} \mathcal{F}_{2}, \mathcal{F}_{3}$ satisfying assumptions of Proposition 3.5 , arcs $\mathcal{F}_{1}, \mathcal{F}_{3}$ and arcs $\mathcal{F}_{2}, \mathcal{F}_{4}$ satisfying assumptions of Proposition 3.6, d a curve from Fig. 7c and $E_{\frac{1}{5}}\left(\mathcal{F}_{1} \cup \mathcal{F}_{2} \cup \mathcal{F}_{3} \cup \mathcal{F}_{4}\right)$

(iii) $\kappa \mathcal{F}_{0}(a)>0$ for every $a \neq p_{0}, \kappa \mathcal{F}_{0}\left(p_{0}\right)=0, \kappa \mathcal{F}_{1}\left(q_{1}\right)=0$ and $\kappa \mathcal{F}_{1}\left(p_{1}\right)>0$,

(iv) the rotation number of $\mathcal{F}_{0}$ is smaller than $\frac{1}{2}$,

(v) $\mathcal{F}_{0}, \mathcal{F}_{1}$ are curved in the different sides at parallel pairs $a_{0}, a_{1}$ close to $p_{0}, p_{1}$ and $q_{0}, q_{1}$, respectively.

Then the set $E_{\lambda}\left(\mathcal{F}_{0} \cup \mathcal{F}_{1}\right)$ has a singular point for every $\lambda \in(0,1)$.

Proposition 3.6 Under the assumptions of Proposition 3.5 if we replace (iv) with

(iv) $\mathcal{F}_{0}, \mathcal{F}_{1}$ are curved in the same side at parallel pairs $a_{0}, a_{1}$ close to $p_{0}, p_{1}$ and $q_{0}, q_{1}$, respectively.

Then the set $E_{\lambda}\left(\mathcal{F}_{0} \cup \mathcal{F}_{1}\right)$ has a singular point for every $\lambda \in(-\infty, 0) \cup(1, \infty)$.

The curvature of a curve at a point is not an affine invariant but the ratio of curvatures at parallel points is an affine invariant. Inflexion points are affine invariants and they are easy to detect. Thus Propositions 3.5 and 3.6 are useful to study singularities of affine $\lambda$-equidistants (see Fig. 7). In Fig. 7a and in Fig. 7b we present arcs which satisfy the assumptions of Propositions 3.5 and 3.6, respectively. Notice that $p_{0}$ and $q_{1}$ are inflexion points. By Proposition 3.5 for all $\lambda \in(0,1)$ an affine $\lambda$-equidistant of a set $\mathcal{F}_{0} \cup \mathcal{F}_{1}$ in Fig. 7a is singular. Similarly, for all $\lambda \in(-\infty, 0) \cup(1, \infty)$ an affine $\lambda$-equidistant of a set $\mathcal{F}_{0} \cup \mathcal{F}_{1}$ in Fig. $7 \mathrm{~b}$ is singular. Furthermore, in Fig. 7c we present $\operatorname{arcs} \mathcal{F}_{1}, \mathcal{F}_{2}, \mathcal{F}_{3}, \mathcal{F}_{4}$ of one curve such that:

- $\operatorname{arcs} \mathcal{F}_{1}, \mathcal{F}_{4}$ and $\operatorname{arcs} \mathcal{F}_{2}, \mathcal{F}_{3}$ satisfy the assumptions of Proposition 3.5,

- $\operatorname{arcs} \mathcal{F}_{1}, \mathcal{F}_{3}$ and $\operatorname{arcs} \mathcal{F}_{2}, \mathcal{F}_{4}$ satisfy the assumptions of Proposition 3.6, 
therefore for all $\lambda \neq 0,1$ an affine $\lambda$-equidistant of a set $\mathcal{F}_{1} \cup \mathcal{F}_{2} \cup \mathcal{F}_{3} \cup \mathcal{F}_{4}$ is singular. In Fig. 7d we illustrate a curve from Fig. 7c together with an affine $\frac{1}{5}$-equidistant of $\mathcal{F}_{1} \cup \mathcal{F}_{2} \cup \mathcal{F}_{3} \cup \mathcal{F}_{4}$.

Proposition 3.7 Let $\mathcal{F}_{0}$ and $\mathcal{F}_{1}$ be embedded regular curves with endpoints $p, q_{0}$ and $p, q_{1}$, respectively. Let $l_{0}$ be the line through $q_{1}$ parallel to $T_{p} \mathcal{F}_{0}$ and let $l_{1}$ be the line through $q_{0}$ parallel to $T_{p} \mathcal{F}_{1}$. Let $c=l_{0} \cap l_{1}, b_{0}=l_{0} \cap T_{p} \mathcal{F}_{1}, b_{1}=l_{1} \cap T_{p} \mathcal{F}_{0}$. Let us assume that

(i) $T_{p} \mathcal{F}_{0} \| T_{q_{1}} \mathcal{F}_{1}$ and $T_{q_{0}} \mathcal{F}_{0} \| T_{p} \mathcal{F}_{1}$,

(ii) the curvature of $\mathcal{F}_{i}$ for $i=0,1$ does not vanish at any point,

(iii) absolute values of rotation numbers of $\mathcal{F}_{0}$ and $\mathcal{F}_{1}$ are the same and smaller than $\frac{1}{2}$,

(iv) for every point $a_{i}$ in $\mathcal{F}_{i}$, there is exactly one point $a_{j}$ in $\mathcal{F}_{j}$ such that $a_{i}, a_{j}$ is a parallel pair for $i \neq j$,

(v) $\mathcal{F}_{0}, \mathcal{F}_{1}$ are curved in the different sides at every parallel pair $a_{0}, a_{1}$ such that $a_{i} \in \mathcal{F}_{i}$ for $i=0,1$.

Let $\rho_{\max }$ (resp. $\rho_{\min }$ ) be the maximum (resp. the minimum) of the set $\left\{\frac{c-b_{1}}{q_{1}-b_{1}}, \frac{c-b_{0}}{q_{0}-b_{0}}\right\}$.

If $\rho_{\max }<1$ then the set $E_{\lambda}\left(\mathcal{F}_{0} \cup \mathcal{F}_{1}\right)$ has a singular point for every $\lambda \in\left[\frac{\rho_{\max }}{\rho_{\max }+1}, \frac{1}{\rho_{\max }+1}\right]$.

If $\rho_{\text {min }}>1$ then the set $E_{\lambda}\left(\mathcal{F}_{0} \cup \mathcal{F}_{1}\right)$ has a singular point for every $\lambda \in\left[\frac{1}{\rho_{\min }+1}, \frac{\rho_{\min }}{\rho_{\min }+1}\right]$.

In particular, if $\rho_{\max }<1$ or $\rho_{\min }>1$ then the Wigner caustic of $\mathcal{F}_{0} \cup \mathcal{F}_{1}$ has a singular point.

Proof Let us consider the case $\rho_{\max }<1$, the proof of the case $\rho_{\min }>1$ is similar.

Since $\mathrm{E}_{\lambda}\left(\mathcal{F}_{0} \cup \mathcal{F}_{1}\right)=\mathrm{E}_{1-\lambda}\left(\mathcal{F}_{0} \cup \mathcal{F}_{1}\right)$, to finish the proof it is enough to show that $\mathrm{E}_{\lambda}\left(\mathcal{F}_{0} \cup \mathcal{F}_{1}\right)$ has a singular point for $\lambda \in\left[\frac{\rho_{\max }}{1+\rho_{\max }}, \frac{1}{2}\right]$.

Let us fix $\lambda$ in the interval $\left[\frac{\rho_{\max }}{1+\rho_{\max }}, \frac{1}{2}\right]$.

We can transform the parallelogram bounded by $T_{p} \mathcal{F}_{0}, T_{q_{0}} \mathcal{F}_{0}, T_{p} \mathcal{F}_{1}, T_{q_{1}} \mathcal{F}_{1}$ to the unit square by an affine transformation $A: \mathbb{R}^{2} \rightarrow \mathbb{R}^{2}$ (see Fig. 8). Since any affine $\lambda$-equidistant is affine equivariant, the sets $\mathrm{E}_{\lambda}\left(A\left(\mathcal{F}_{0} \cup \mathcal{F}_{1}\right)\right)$ and $A\left(\mathrm{E}_{\lambda}\left(\mathcal{F}_{0} \cup \mathcal{F}_{1}\right)\right)$ coincide. Let us consider the coordinate system described in Fig. $8 \mathrm{~b}$.

Let $\ell_{i}$ be the length of $\mathcal{F}_{i}^{\prime}$ for $i=0,1$. Let $\left[0, \ell_{0}\right] \ni s \mapsto f(s)=\left(f_{1}(s), f_{2}(s)\right)$ and $\left[0, \ell_{1}\right] \ni t \rightarrow g(t)=\left(g_{1}(t), g_{2}(t)\right)$ be arc length parameterizations of $\mathcal{F}_{0}^{\prime}=A\left(\mathcal{F}_{0}\right)$ and $\mathcal{F}_{1}^{\prime}=A\left(\mathcal{F}_{1}\right)$, respectively, such that:

(a) $f(0)=g\left(\ell_{1}\right)=(0,0), g(0)=\left(g_{1}(0), 1\right)$, where $0<g_{1}(0) \leqslant \rho_{\max }$ and $f\left(\ell_{0}\right)=\left(1, f_{2}\left(\ell_{0}\right)\right)$, where $0<f_{2}\left(\ell_{0}\right) \leqslant \rho_{\max }$,

(b) $\frac{d f}{d s}(0)=[1,0], \frac{d f}{d s}\left(\ell_{0}\right)=[0,1], \frac{d g}{d t}(0)=[-1,0], \frac{d g}{d t}\left(\ell_{1}\right)=[0,-1]$.

From the implicit function theorem there exists a smooth function $t:\left[0, \ell_{0}\right] \rightarrow$ $\left[0, \ell_{1}\right]$ such that

$$
\frac{d f}{d s}(s)=-\frac{d g}{d t}(t(s))
$$



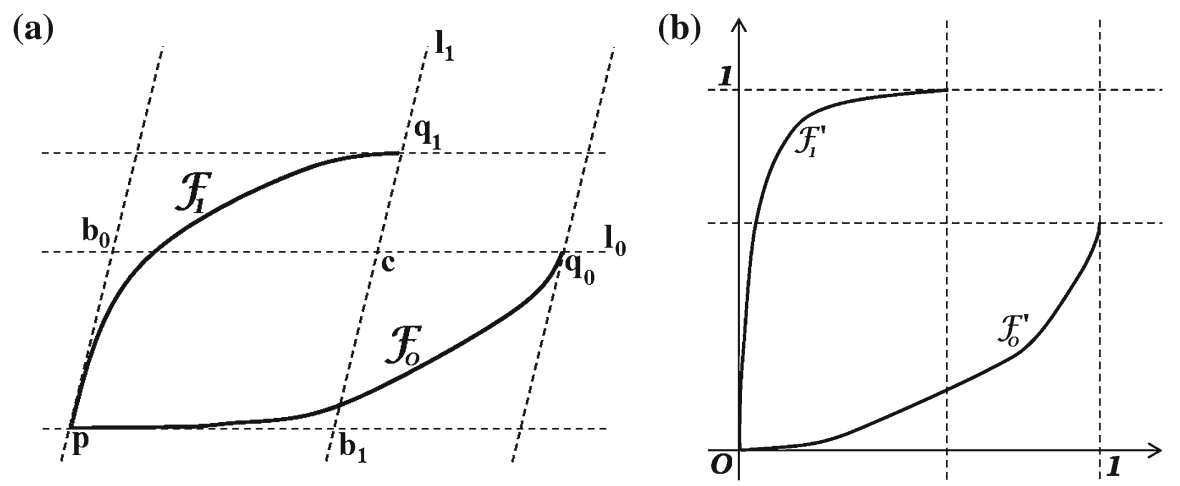

Fig. 8 a Arcs satisfying assumptions of Proposition 3.7, $\mathbf{b}$ the coordinate system used in the proof of Proposition 3.7

It means that $f(s), g(t(s))$ is a parallel pair.

Let $\varrho=\frac{\lambda}{1-\lambda}$. Then $\varrho \in\left[\rho_{\max }, 1\right]$. By Proposition 2.5 the set $\mathrm{E}_{\lambda}\left(\mathcal{F}_{0}^{\prime} \cup \mathcal{F}_{1}^{\prime}\right)$ is singular if $\frac{\kappa_{\mathcal{F}_{0}^{\prime}}(f(s))}{\kappa_{\mathcal{F}_{1}^{\prime}}(g(t(s)))}=\varrho$ for some $s \in\left[0, \ell_{0}\right]$.

By (3.2) we get that $t^{\prime}(s)=\frac{\kappa_{\mathcal{F}_{0}^{\prime}}(f(s))}{\kappa_{\mathcal{F}_{1}^{\prime}}(g(t(s)))}$. We will show that $t^{\prime}(s)=\varrho$ for some $s \in\left[0, \ell_{0}\right]$. Let us assume that $t^{\prime}(s) \neq \varrho$ for all $s \in\left[0, \ell_{0}\right]$.

By (3.2) we get that

$$
g\left(\ell_{1}\right)-g(0)=\int_{0}^{\ell_{1}} \frac{d g}{d t}(t) d t=\int_{0}^{\ell_{0}} t^{\prime}(s) \frac{d g}{d t}(t(s)) d s=-\int_{0}^{\ell_{0}} t^{\prime}(s) \frac{d f}{d s}(s) d s .
$$

Let us assume that $t^{\prime}(s)>\varrho$ for all $s \in\left[0, \ell_{0}\right]$.

At the first component of (3.3) we have

$$
g_{1}(0)=\int_{0}^{\ell_{0}} t^{\prime}(s) \frac{d f_{1}}{d s}(s) d s>\int_{0}^{\ell_{0}} \varrho \cdot \frac{d f_{1}}{d s}(s) d s=\varrho .
$$

Then $g_{1}(0)>\varrho$ which is impossible, since $g_{1}(0) \leq \rho_{\max }$ and $\varrho \in\left[\rho_{\max }, 1\right]$.

If we assume that $t^{\prime}(s)<\varrho$ for all $s \in\left[0, \ell_{0}\right]$, then in a similar way, we get $1<\varrho^{2}$, which also is impossible, since $\varrho \in\left[\rho_{\max }, 1\right]$ and $\rho_{\max }>0$.

Therefore there exists $s \in\left[0, \ell_{0}\right]$ such that $t^{\prime}(s)=\varrho$ which ends the proof.

In the similar way we get the two following propositions.

Proposition 3.8 Let $\mathcal{F}_{0}$ and $\mathcal{F}_{1}$ be embedded regular curves with endpoints $p, q_{0}$ and $p, q_{1}$, respectively. Let $l_{0}$ be the line through $q_{0}$ parallel to $T_{p} \mathcal{F}_{0}, l_{1}$ be the line through $q_{1}$ parallel to $T_{p} \mathcal{F}_{1}$ and $l_{p}$ be the line through p parallel to $T_{q_{0}} \mathcal{F}_{0}$. Let $c=l_{0} \cap T_{q_{1}} \mathcal{F}_{1}$, $b_{0}=l_{0} \cap l_{p}$ and $b_{1}=T_{p} \mathcal{F}_{1} \cap T_{q_{1}} \mathcal{F}_{1}$. Let us assume that

(i) $T_{p} \mathcal{F}_{0}=T_{p} \mathcal{F}_{1}$ and $T_{q_{0}} \mathcal{F}_{0} \| T_{q_{1}} \mathcal{F}_{1}$, 
Fig. 9 Arcs satisfying assumptions of Proposition 3.8

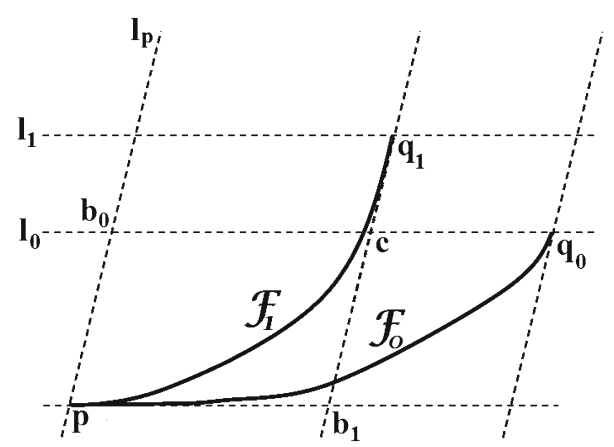

(ii) the curvature of $\mathcal{F}_{i}$ for $i=0,1$ does not vanish at any point,

(iii) absolute values of rotation numbers of $\mathcal{F}_{0}$ and $\mathcal{F}_{1}$ are the same and smaller than $\frac{1}{2}$,

(iv) for every point $a_{i}$ in $\mathcal{F}_{i}$, there is exactly one point $a_{j}$ in $\mathcal{F}_{j}$ such that $a_{i}, a_{j}$ is a parallel pair for $i \neq j$,

(v) $\mathcal{F}_{0}, \mathcal{F}_{1}$ are curved in the same side at every parallel pair $a_{0}, a_{1}$ such that $a_{i} \in \mathcal{F}_{i}$ for $i=0,1$.

Let $\rho_{\max }$ (resp. $\rho_{\min }$ ) be the maximum (resp. the minimum) of the set $\left\{\frac{c-b_{1}}{q_{1}-b_{1}}, \frac{c-b_{0}}{q_{0}-b_{0}}\right\}$.

If $\rho_{\max }<1$ then the set $E_{\lambda}\left(\mathcal{F}_{0} \cup \mathcal{F}_{1}\right)$ has a singular point for every $\lambda \in\left(-\infty,-\frac{\rho_{\max }}{1-\rho_{\max }}\right] \cup\left[\frac{1}{1-\rho_{\max }}, \infty\right)$.

If $\rho_{\min }>1$ then the set $E_{\lambda}\left(\mathcal{F}_{0} \cup \mathcal{F}_{1}\right)$ has a singular point for every $\lambda \in\left(-\infty, \frac{1}{1-\rho_{\min }}\right] \cup\left[-\frac{\rho_{\min }}{1-\rho_{\min }}, \infty\right)$.

Arcs satisfying assumptions of Proposition 3.8 are illustrated in Fig. 9.

Proposition 3.9 Let $\mathcal{F}_{0}, \mathcal{F}_{1}$ be embedded curves with endpoints $p, q_{0}$ and $p, q_{1}$, respectively. Let $l$ be the line through $p$ and $q_{1}$ and let $c=l \cap T_{q_{0}} \mathcal{F}_{0}$. Let us assume that

(i) $T_{p} \mathcal{F}_{0}\left\|T_{q_{0}} \mathcal{F}_{0}\right\| T_{p} \mathcal{F}_{1} \| T_{q_{1}} \mathcal{F}_{1}$,

(ii) the curvature of $\mathcal{F}_{i}$ does not vanish for $i=0,1$,

(iii) the absolute value of the rotation number of $\mathcal{F}_{i}$ is equal to $\frac{1}{2}$ for $i=0,1$.

Let $\rho=\left|\frac{q_{1}-p}{c-p}\right|$. If $\mathcal{F}_{0}, \mathcal{F}_{1}$ are curved in the different sides at every parallel pair $a_{0}, a_{1}$ such that $a_{0} \in \mathcal{F}_{0}, a_{1} \in \mathcal{F}_{1}$ then the set $E_{\lambda}(\mathcal{F} \cup \mathcal{G})$ has a singular point for $\lambda \in\left\{\frac{1}{1+\rho}, \frac{\rho}{1+\rho}\right\}$.

If $\rho \neq 1$ and $\mathcal{F}_{0}, \mathcal{F}_{1}$ are curved in the same side at every parallel pair $a_{0}, a_{1}$ such that $a_{0} \in \mathcal{F}_{0}, a_{1} \in \mathcal{F}_{1}$ then the set $E_{\lambda}\left(\mathcal{F}_{0} \cup \mathcal{F}_{1}\right)$ has a singular point for $\lambda \in\left\{\frac{-1}{\rho-1}, \frac{\rho}{\rho-1}\right\}$. 
Fig. 10 Arcs $\mathcal{F}_{0}, \mathcal{F}_{1}$, the Wigner caustic of $\mathcal{F}_{0} \cup \mathcal{F}_{1}$ and the tangent lines to $\mathcal{F}_{0}$ and to $\mathcal{F}_{1}$ (the dashed ones)

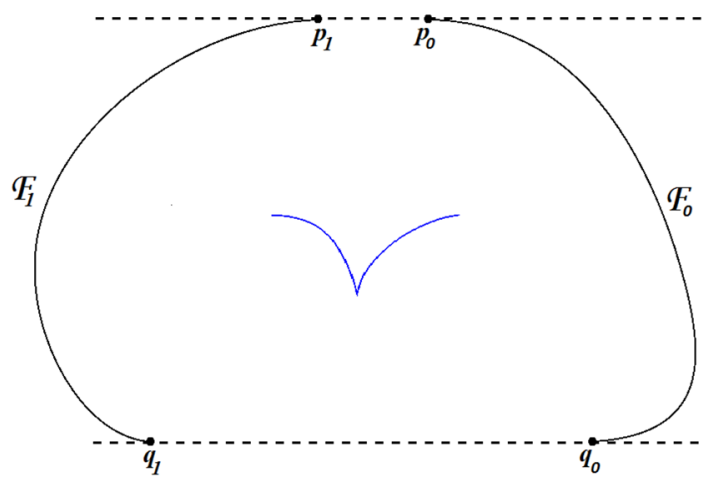

(a)

(b)
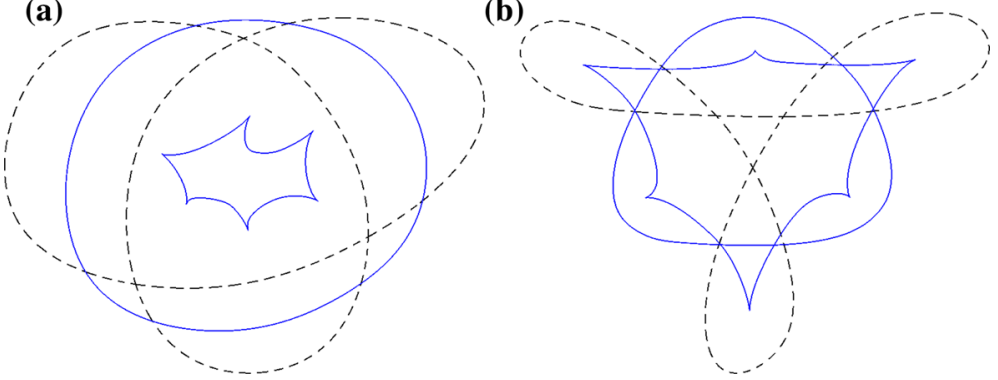

Fig. 11 A smooth regular curves with non-vanishing curvature (the dashed lines) and their Wigner caustics
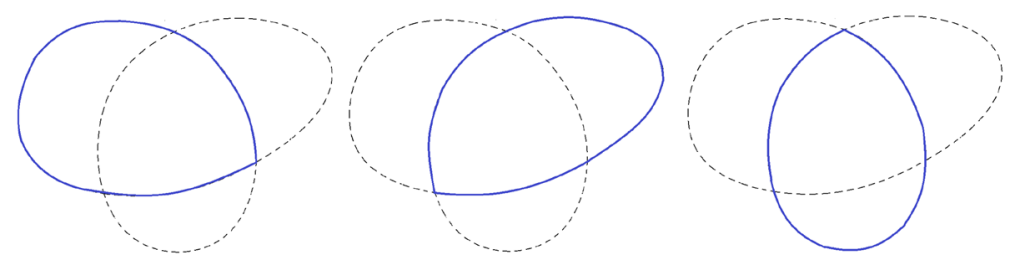

Fig. 12 Convex (the continuous line) and non-convex (the dashed line) loops of a curve presented in Fig. $11 \mathrm{a}$

(a)

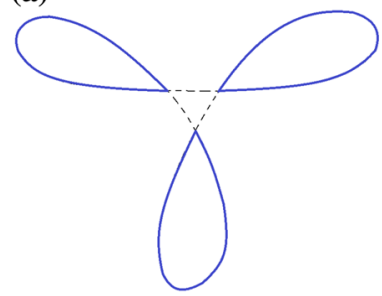

(b)

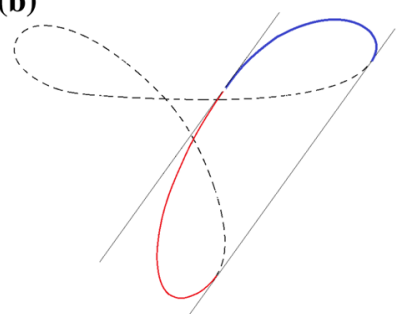

Fig. 13 a Convex loops (the continuous line) of a curve presented in Fig. 11b, b arcs of a curve (the continuous lines) which satisfy the assumptions of Proposition 3.9 
Remark 3.10 Let $M$ be a smooth closed regular curve. If there exists $p$ in $\mathbb{R}^{2}$ and arcs $\mathcal{F}, \mathcal{G}$ of $M$ such that $\mathcal{F}, \tau_{p}(\mathcal{G})$ fulfill the assumptions of one of Propositions 3.7,3.8 or 3.9 , where $\tau_{p}$ is the translation by $p$, then the affine $\lambda$-equidistant of $M$ has a singular point for $\lambda$ described in the above propositions.

Let us notice that curves $\mathcal{F}_{0}$ and $\tau_{p_{0}-p_{1}}\left(\mathcal{F}_{1}\right)$ in Fig. 10 satisfy Proposition 3.9. In that case by Remark 3.10 the Wigner caustic of $\mathcal{F}_{0} \cup \mathcal{F}_{1}$ has a singular point.

Since the curve presented in Fig. 11a has exactly three convex and three non-convex loops (see Fig. 12), its Wigner caustic has at least 6 singular points by Theorem 3.2. The curve presented in Fig. 11b has exactly three convex loops (see 13a) so its Wigner caustic has at least 3 singular points. Despite the fact that this curve has no non-convex loops we can apply Proposition 3.9 and get that the Wigner caustic of this curve has at least 3 extra singular points (see Fig. 13b). Hence the Wigner caustic of this curve has at least 6 singular points.

Open Access This article is distributed under the terms of the Creative Commons Attribution 4.0 International License (http://creativecommons.org/licenses/by/4.0/), which permits unrestricted use, distribution, and reproduction in any medium, provided you give appropriate credit to the original author(s) and the source, provide a link to the Creative Commons license, and indicate if changes were made.

\section{References}

Arnold, V.I.: Singularities of Caustics and Wave Fronts, vol. 62. Kluwer, South Holland (2001). (Math. and its Appl.)

Berry, M.V.: Semi-classical mechanics in phase space: a study of Wigner's function. Philos. Trans. R. Soc. Lond. A 287, 237-271 (1977)

Craizer, M., Domitrz, W., de Rios, P.M.: Even dimensional improper affine spheres. J. Math. Anal. Appl. 421, 1803-1826 (2015)

Cufi, J., Gallego, E., Reventós, A.: A note on Hurwitz's inequality. J. Math. Anal. Appl. 458, 436-451 (2018)

Domitrz, W., Janeczko, S., de Rios, P.M., Ruas, M.A.S.: Singularities of affine equidistants: extrinsic geometry of surfaces in 4-space. Bull. Braz. Math. Soc. 47(4), 1155-1179 (2016)

Domitrz, W., Manoel, M., de Rios, P.M.: The Wigner caustic on shell and singularities of odd functions. J. Geom. Phys. 71, 58-72 (2013)

Domitrz, W., de Rios, P.M.: Singularities of equidistants and global centre symmetry sets of Lagrangian submanifolds. Geom. Dedicata 169, 361-382 (2014)

Domitrz, W., de Rios, P.M., Ruas, M.A.S.: Singularities of affine equidistants: projections and contacts. J. Singul. 10, 67-81 (2014)

Domitrz, W., Romero Fuster, M.C., Zwierzyński, M.: The geometry of the Secant caustic of a planar curve. arXiv: 1803.00084

Domitrz, W., Zwierzyń ski, M.: The geometry of the Wigner caustic and affine equidistants of planar curves. arXiv:1605.05361v4

Domitrz, W., Zwierzyński, M.: The Gauss-Bonnet theorem for coherent tangent bundles over surfaces with boundary and its applications. arXiv: 1802.05557

Giblin, P.J., Holtom, P.A.: The Centre Symmetry Set, Geometry and Topology of Caustics, vol. 50, pp. 91-105. Banach Center Publications, Warsaw (1999)

Giblin, P.J., Reeve, G.M.: Centre symmetry sets of families of plane curves. Demonstr. Math. 48, 167-192 (2015)

Giblin, P.J., Warder, J.P., Zakalyukin, V.M.: Bifurcations of affine equidistants. Proc. Steklov Inst. Math. 267, 57-75 (2009)

Giblin, P.J., Zakalyukin, V.M.: Singularities of centre symmetry sets. Proc. London Math. Soc. 90, 132-166 (2005). (3) 
Góźdź, S.: An antipodal set of a periodic function. J. Math. Anal. Appl. 148, 11-21 (1990)

Janeczko, S.: Bifurcations of the center of symmetry. Geom. Dedicata 60, 9-16 (1996)

Janeczko, S., Jelonek, Z., Ruas, M.A.S.: Symmetry defect of algebraic varieties. Asian J. Math. 18(3), 525-544 (2014)

Laugwitz, D.: Differential and Riemannian Geometry. Academic Press, New York (1965)

Ozorio de Almeida, A.M., Hannay, J.: Geometry of two dimensional Tori in phase space: projections, sections and the Wigner function. Ann. Phys. 138, 115-154 (1982)

Schneider, R.: Reflections of planar convex bodies. In: Adiprasito, K., Barany, I., Vilcu, C. (eds.) Convexity and Discrete Geometry Including Graph Theory, Mulhouse, France, September 2014, pp. 69-76, Springer, (2016)

Schneider, R.: The middle hedgehog of a planar convex body. Beitr. Algebra Geom. 58, 235-245 (2017)

Zakalyukin, V.M.: Envelopes of families of wave fronts and control theory. Proc. Steklov Math. Inst. 209, 133-142 (1995)

Zwierzyński, M.: The improved isoperimetric inequality and the Wigner caustic of planar ovals. J. Math. Anal. Appl 442(2), 726-739 (2016)

Zwierzyński, M.: The Constant Width Measure Set, the Spherical Measure Set and isoperimetric equalities for planar ovals. arXiv:1605.02930

Publisher's Note Springer Nature remains neutral with regard to jurisdictional claims in published maps and institutional affiliations. 